\title{
Development of Synchronizer Operation for integration in AMT Control Strategy
}

\author{
N.R.Junnarkar,V.N.Raibhole \\ (Dept. of Mechanical Engineering, M.E.S. College of Engineering, S.P.University of Pune, India)
}

\begin{abstract}
$\overline{\text { Abstract: We see many developments in the vehicles and their transmission systems. These majorly focus on the }}$ latest market trends, user demands and better sophisticated technology. The transmission system developer must closely observe the market and the changing values in the society as the transmission system doesn't change in an overnight. They require sufficient time to get established and become stable in the market.The scope of the paper involves creating the control strategy for the working of the synchronizer for an AMT vehicle in MATLAB Simulink. In MATLAB Simulink a mathematical working model of the component is developed. The output of the model estimates the force of actuation and the time required during the shift process which predicts the actuator parameters, used further to operate the actuators. Once the mathematical model of control strategy is defined and its values are obtained, these can be implemented in the vehicle design model.
\end{abstract}

Keywords - Synchronizer, Actuation Force, Synchronization Time, AMT Control Strategy, Gear Shifting

\section{INTRODUCTION}

The recent trends in automobile industry aims at making the vehicle efficient, easy to drive and control. Modern automobiles demand for fast speeds and high torques that require high gear shifting forces. Hence, for the ease of gear shifting, the synchronizers must be efficient and have higher capacities.Various transmission methods that basically involve automating the vehicle (fully or partially) are emerging aiming at achievement of these aims. The automation reduces the driver effort in gear shift thus making driving the vehicle comfortable and easy. The knowledge of the synchronizer control strategy, its various forces and the synchronization timings are a must for automating the vehicle. With the aim of designing and developing the mathematical model of a synchronizer and a clutch for the AMT application many technical papers were referred. The aim for the reference in the first stage was to understand the working of the synchronizer, its function in an automobile and various mathematical equations defining the synchronizer working. The basic idea of synchronizer, its application, the performance characteristics as well as its features and limitations are discussed by U. Wazir [1].the future trends are also addressed along with a brief calculation of the synchronizer.Muralidharan, S. And Prasad, S. [2] have discussed the current and future necessities of increasing the fuel economy and reducing the emissions. Further the problems associated with driving in crowded cities like stress to the driver due to constant changing of gears and clutch operation have led to the emergence of automatic transmission. The automatic transmission has efficiency lower than the manual; hence the best alternative to solve both the problems is automation of the manual transmission.D.V Neto, D. G Florencio, P Rodrigues and J Fernandez [3] have described the Manual transmission synchronizer, its function and design. The mutual working of the gear shift lever working and transmission components is discussed. The synchronizers have been used for quite along. With the increase in the torque ratings of the engine and higher torque demands, improvements are done in the transmission system. These also include modification in the pre-existing synchronizers. Pingale, A. [4] has discussed the conventional synchronizer, its components and design. Further he has suggested new modifications to overcome the existing challenges of the conventional synchronizers. Razzacki, S [5] has put forth detailed calculation and design method for a synchronizer. For establishing the sleeve and blocker pointing angle- relationship with the synchronizer size, frictional coefficient, cone and index torque, a mathematical algorithm is developed. This relationship is also represented graphically so as to identify the clash and hard shift zones. Luo, F., Li, Feng, X., and Zhang, Y [6] have presented the model of a self-energizing synchronizer along with its simulation model. The synchronizer performance is analyzed using ADAMS. Based on the model the behavior of the synchronizer with asymmetric arrangement of chamfer teeth and effect of the stiffness of the energizing spring are studied.The need for automation is a necessity in the recent times, for it the synchronization time and various actuation forces are required. This paper involves a stepwise mathematical approach of finding these values. A particular synchronizer model is considered for which the input parameters are known. The mathematical model developed involves all the factors necessary for computing the values depending upon the inputs given. This strategy is then embedded in the AMT model for the automation purpose. 


\section{DESCRIPTION OF AUTOMOTIVE SYNCHRONIZER:}

Synchronizers are in the form of friction clutches that have the main function of synchronizing the rotational speed of the required gear with the output transmission shaft. The rotational speeds of the two are to be synchronized before their engagement so as to allow a smooth shift. Synchronizers are found in different types depending upon their application; but their basic function remains the same.For defining the control strategy of the synchronizer and calculating various actuating torques and forces, the synchronizer basics should be studied.

\subsection{BASICS OF SYNCHRONIZER}

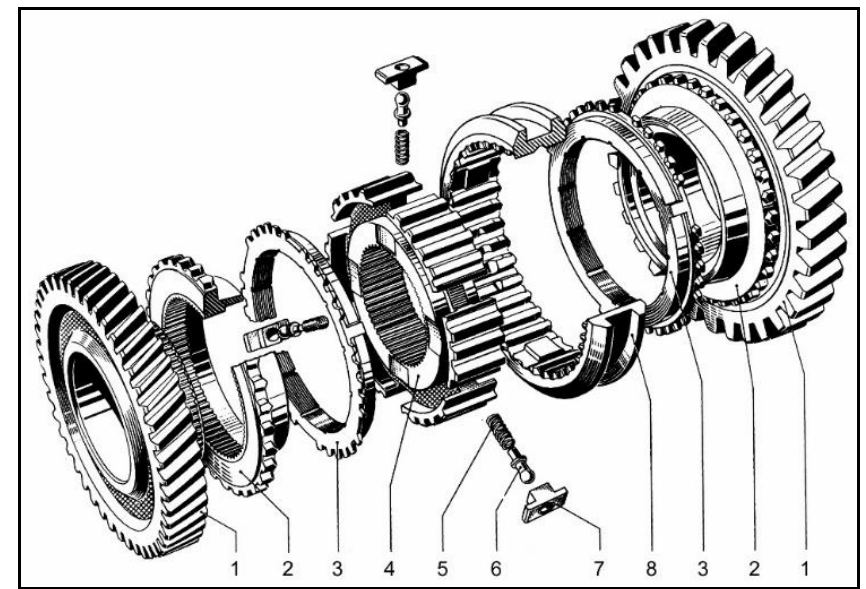

Fig.1: Synchronizer Components (Source: Automotive Transmission pg.316)

The major components of an automobile synchronizer and their functions are discussed in brief in Fig.1:

1- Gear Wheel

2- Gear Cones: they have external teeth that mesh with the internal teeth of the shift sleeve to synchronize the gear speed with that of the hub.

3- Blocker Ring: it produces the synchronization torque required for synchronizing the input and output shafts by the inter locking of its external teeth with the internal teeth of the sliding sleeve.

4- Synchronizer Hub: is connected rigidly to the input or output shaft using splines

5- Energizing Spring

6- Ball Pin

7- Strut: it generates load on the synchronization ring to perform the pre-synchronization process. It's situated in the synchronizer body, between the shift sleeve inner groove and the hub.

8- Gearshift Sleeve: the internal splines are in constant mesh with the synchronizer external splines, so as to move only axially. It has allocation for the gear shift fork on its outer periphery.

\subsection{SyNChronization Process}

The process of synchronization takes place in eight stages. A brief over view of the synchronization process is discussed below. The Fig. 2 shows the stepwise occurrence of the synchronization process.

Stage 0 Neutral Position (Sleeve in neutral position)

Stage 1 Pre-synchronization (sleeve moves ahead and touches the outer ring that rotates in order to stop the sleeve movement)

Stage 2 Start boost (sleeve touches the outer ring, the blocking teeth prevent sleeve movement. Claw plate rotates and friction torque builds up)

Stage 3 Synchronization (friction torque increases to the largest and relative force between the clutch gear and hub is zero)

Stage 4 Blocking Release (shift force acts on the blocker ring. As the speeds equalize, index torque overcomes friction torque and the sleeve rotates to frictionally engage the blocker ring and gear. Shift sleeve teeth slide on blocker ring chamfers)

Stage 5 Free Flight (the blocker ring indexes the sleeve so that it moves further and mesh the gear. As the sleeve easily engages, the shifting force drops)

Stage 6 Bump (the sleeve teeth and gear dog teeth hit and the sleeve again gets blocked. A bump is produced with the contact of the sleeve teeth and clutch gear teeth) 
Stage 7 Engagement (the clutch gear teeth get engaged with the clutch gear as the slip teeth rotates the clutch gear teeth. Here the synchronization begins)

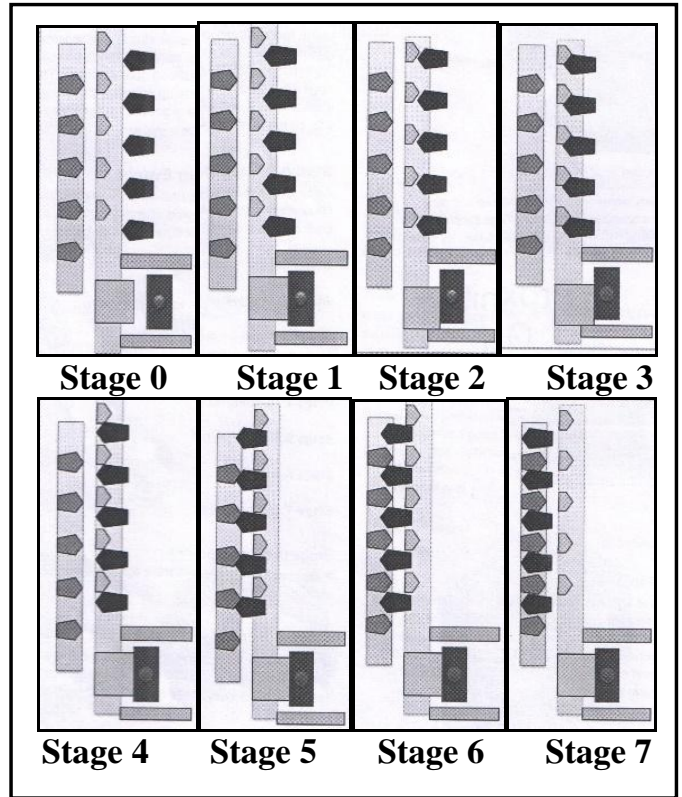

Fig. 2: Phases of Synchronization

\section{MATHEMATICAL MODEL:}

For defining the control strategy of the synchronizer, a mathematical model is developed in MATLAB Simulink. Various input parameters are fed into the model. The forces that act on the synchronizer during its functioning are considered, computed and given as an input into the model. The model gives the values of synchronization time for various gear shifts in both cases of up-shift and down-shift as an output.

\subsection{Parameters To Be CONSIDERed:}

\section{A. BREAK THROUGH LOAD (BTL):}

The Break Through Load (BTL) causes the blocker ring into block position. When the force is applied at the sleeve, the BTL should move the sleeve and stay until the sleeve and blocker ring tooth meet. If the BTL gets with drawn too fast, it causes the gears to clash and if it stays for a prolonged period, it causes the ring to stick. BTL can be calculated using physics and the free body diagram of the ball strut and detent groove in the sleeve.

$$
\begin{aligned}
& F_{A}=F_{R} \frac{\sin \theta+\mu \cos \theta}{\cos \theta-\mu \sin \theta} \\
& F_{R}=N_{S}(\cos \theta-\mu \sin \theta) \\
& B T L=3 F_{A}
\end{aligned}
$$

Here,

$\mathrm{F}_{\mathrm{A}}$ : Axial force for overcoming spring reaction

$\mathrm{F}_{\mathrm{R}}$ : Reaction Force (detent)

\section{B. CONE TORQue $\left(\mathbf{T}_{\mathrm{C}}\right)$ :}

Friction is generated in the cone angle direction, between the cone and the friction surfaces due to the blocker ring. Cone angle is a function of the cone angle, friction of the surfaces, axial force acting on the sleeve and the cone diameter. It's calculated as:

$$
T_{C}=\frac{F x R x \mu_{c}}{\sin \alpha}
$$




\section{INDEX TORQUE $\left(\mathbf{T}_{\mathrm{I}}\right)$ :}

The indexed torque indexes the blocker ring and moves the sleeve. Friction is generated as the sleeve pointing contacts the blocker ring. This friction generates the torque called Index Torque. Index Torque is a function of sleeve axial force, tooth pointing angle, surface friction between the ring and sleeve. It's calculated as:

$$
\begin{gathered}
T_{1}=F_{1} * r \\
F=N_{S}\left(\sin \beta-\mu_{P} \cos \beta\right) \\
F_{1}=F \frac{\cos \beta-\mu \sin \beta}{\sin \beta+\mu \cos \beta}
\end{gathered}
$$

\section{SYNCHRONIZATION TIME $\left(\mathbf{T}_{\mathrm{SYN}}\right)$ :}

Synchronization time gives the time the synchronizer requires to equalize the speeds of the input and output shafts. The synchronization time depends on the cone torque, speed difference of the gears and inertia. It can be mathematically given as:

$T_{S Y N}=\frac{I x 2 \pi x \square \omega}{60 x\left(T_{C}+T_{D}\right)}$

The component $\mathrm{T}_{\mathrm{D}}$ here is called the 'Drag Torque or Torque loss'. It depends on Torque due to Fluid churning and friction torque. From the literature survey its value is take as $1 \mathrm{~N}$.

The time from equation (8) is obtained in seconds. This time decides when the actuator engages the clutch again i.e. the time for the shift of gear in an automatic vehicle.

\subsection{INPUTS FOR THE MATHEMATICAL MODEL:}

The synchronizer was mathematically modelled using MATLAB Simulink for the purpose of finding the synchronization time and its actuating forces. The dimensional aspects or force / torque considerations were got either from the 3D scanned modelled of the pre-existing synchronizer or via literature survey.For an engine of 2500rpm the synchronizer considered for the modelling purpose has the following parameters:

Table.1: The Parameters of the synchronizer model considered

\begin{tabular}{|l|l|}
\hline \multicolumn{1}{|c|}{ Component } & Specification \\
\hline Pointing angle $(\beta):$ & $67^{\circ}$ \\
\hline Sleeve detent ramp angle $(\theta)$ & $60^{\circ}$ \\
\hline Cone angle $(\alpha)$ : & $7^{\circ}$ \\
\hline $\begin{array}{l}\text { Coefficient of friction sleeve } \\
\text { through out }\end{array}$ & 0.11 \\
\hline Sleeve / ring diameter $(\mathrm{dp})$ & $0.0925 \mathrm{~m}$ \\
\hline Cone gauge diameter $(\mathrm{d})$ & $0.0768 \mathrm{~m}$ \\
\hline Engine Inertia $(\mathrm{I})$ & $0.42 \mathrm{~kg} / \mathrm{m} 2$ \\
\hline Transmission Input Inertia $(\mathrm{I})$ & $0.01 \mathrm{~kg} / \mathrm{m} 2$ \\
\hline
\end{tabular}

The equations of the forces and torques acting on the synchronizer are modelled in the MATLAB Simulink. The inputs required for the purpose of computation are as given in Table: 1 . Various forces or torques acting upon the synchronizer were calculated and given as an input to the calculation of the synchronization time. The model has a provision for both up-shift and down-shift of gears, along with that for the respective gear changes in each shift. The synchronization time changed for each case as the gear speeds changed.The mathematical model for the Synchronizer is made in MATLAB Simulink is as in Fig.3. 


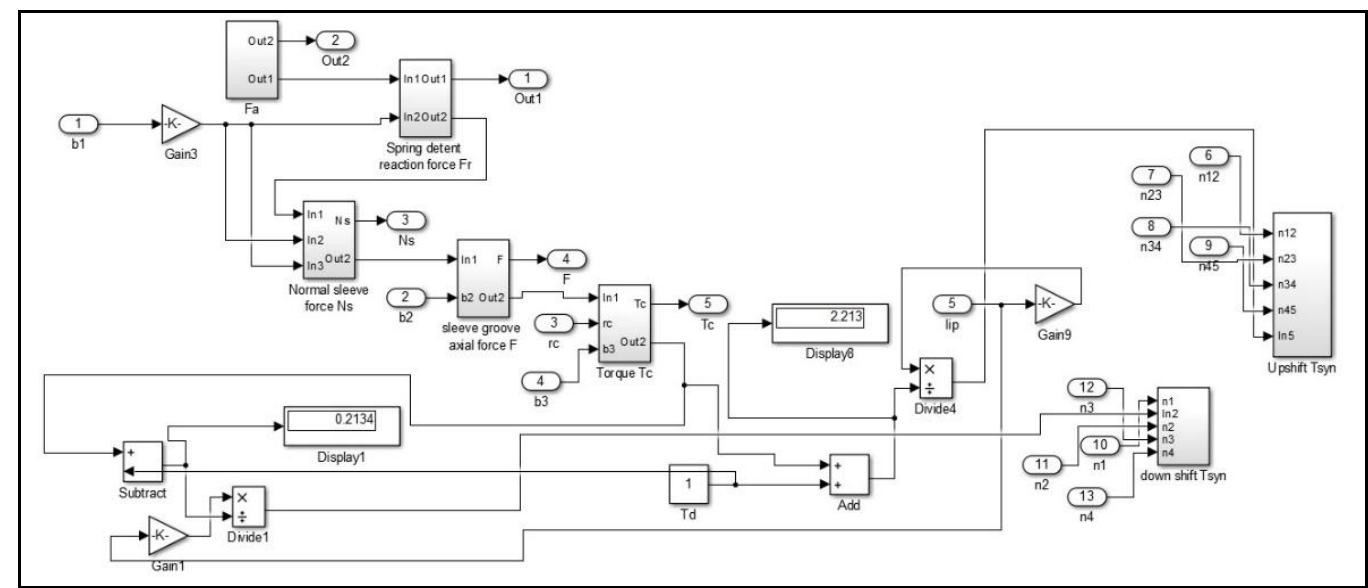

Fig. 3: MATLAB Model for Synchronizer

\section{RESULTS AND DISCUSSION:}

The Break Through Load (BTL) is taken as $45 \mathrm{~N}$ from literature. Depending upon the BTL, the input parameters (Table: 1) and equations in section 3.1various results were obtained from the mathematical model. The Table. 2 summarizes the obtained actuation forces and torques.

Table.2: Actuation Forces and Torques

\begin{tabular}{|l|l|}
\hline \multicolumn{1}{|c|}{ Parameter } & \multicolumn{1}{|c|}{ Value } \\
\hline Break Through Load $(\mathrm{BTL})$ & $45 \mathrm{~N}$ \\
\hline $\begin{array}{l}\text { Axial Force to overcome detent } \\
\text { spring action }\left(\mathrm{F}_{\mathrm{A}}\right)\end{array}$ & $15 \mathrm{~N}$ \\
\hline Spring Reaction Force $\left(\mathrm{F}_{\mathrm{R}}\right)$ & $6.6 \mathrm{~N}$ \\
\hline Normal Sleeve Force $\left(\mathrm{N}_{\mathrm{s}}\right)$ & $16.3 \mathrm{~N}$ \\
\hline Axial force of Sleeve Groove $(\mathrm{F})$ & $15.7048 \mathrm{~N}$ \\
\hline Index Torque $\left(\mathrm{T}_{\mathrm{I}}\right)$ & $4.45 \mathrm{~N}$ \\
\hline Cone Torque $\left(\mathrm{T}_{\mathrm{c}}\right)$ & $0.544 \mathrm{~N} / \mathrm{m}$ \\
\hline
\end{tabular}

Using the actuation torque, inertia and speed difference values for the synchronizer the synchronization time can be calculated for the gear shifts (up shift and down shift).Table.3 gives the synchronization time results as obtained from the MATLAB Simulink model.

Table.3: Synchronization Time

\begin{tabular}{|c|l|l|l|}
\hline Upshift & Time (sec) & Downshift & Time (sec) \\
\hline $1-2$ & 0.2043 & $2-1$ & 1.271 \\
\hline $2-3$ & 0.3019 & $3-2$ & 1.879 \\
\hline $3-4$ & 0.877 & $4-3$ & 2.413 \\
\hline $4-5$ & 0.2119 & $5-4$ & 1.319 \\
\hline
\end{tabular}

The relationship between the synchronizer time and the synchronization torque (or Cone Torque) is important in synchronizer sizing. We can also determine the synchronizer torque using the equations of motion, given the synchronization time that majorly depends on the initial velocity of synchronizer and gear step. The Fig.4 gives an over view of the relationship between the synchronization time and gear shift for the up-shift and down-shift cases. We can the time for down-shift is more than that for the up-shift as the total drag that causes the input to slow assists the upshift and resists the downshift. This relation can be seen very well from equation (8) in section 3.1 


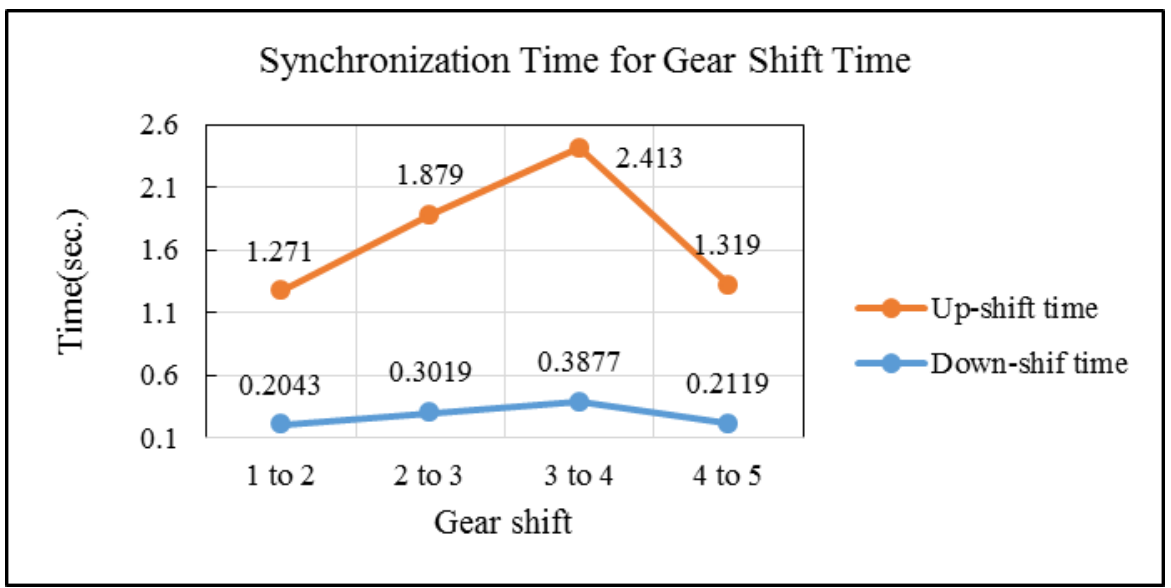

Fig.4: Synchronization Time graph for Gear-shift

\section{CONCLUSION}

In this paper the basics of the synchronizer performance are discussed. The control strategy of the synchronizer for all the gear shifts during both up-shift and down-shift is developed using MATLAB Simulink. This control strategy calculates the synchronization time, actuation forces and actuation torque. The synchronization time is as seen in Table.3. The actuation torque (Cone Torque) calculated is $0.544 \mathrm{~N} / \mathrm{m}$. the other force or torque values can be seen in table.2 The synchronization time is studied with respect to the gear shifts and forces. Depending upon it the graphs (Fig.4) are plotted which show the change in synchronization timings. The actuation torque, force values and synchronization time values, along with the synchronizer control strategy can be embedded in the ECU of the AMT for the gear shifting. The automatic shifting would thus reduce the driver efforts and make driving easy and convenient.

\section{REFERENCES}

[1] U.Wazir,Manual Gearbox Synchronizers-An Overview, Int.Journal of Emerging trends in Engineering and Development,5(3),September 2013,422-428.

[2] S.Muralidharan,S..A.N.Prasad, Design Of Shift Select Mechanism For Automation Of Manual Transmission, SAE Technical Paper ,2014-01-1749,2014, doi:10.4271/2014-01-1749

[3] D. V. Neto, D. G. Florencio, Pedro Rodrigues,Josevaldo Fernandez,Manual Transmission: Synchronization Main Aspects, SAE paper(2006-01-2519), 2006, doi:10.4271/2006-01-2519.

[4] Pingale, A., Development of Compact Synchronizer Technology for Manual and Automated Transmission, SAE Technical Paper 2014-01-1719,2014, doi:10.427172014-01-1719

[5] R.T.Syed T,Synchroniser Design: A Mathematical and Dimensional Treatise, (2004), SAE paper (2004-01-1230)

[6] Luo, F., Li, Feng, X., and Zhang, Y.,Simulation and Analysis on a Self-Energizing Synchronizer of Transmission, SAE Technical Paper 2015-01-0633, 2015, doi: 10.4271/2015-01-0633

[7] D. A. Crolla, Automotive Engineering Powertrain, Chassis System and Vehicle Body,,First edition 2009,Elsevier Inc,USA 